\title{
Costo-efectividad de dabigatrán comparado con warfarina para el tratamiento de pacientes con fibrilación auricular no valvular
}

\author{
Juan J. Triana, Camilo Castañeda, Ludy Parada, Margarita Otálora-Esteban \\ y Diego Rosselli*
}

Departamento de Epidemiología Clínica y Bioestadística, Facultad de Medicina, Pontificia Universidad Javeriana, Bogotá, Colombia

Recibido el 16 de febrero de 2015; aceptado el 1 de junio de 2015

Disponible en Internet el 29 de agosto de 2015

\author{
PALABRAS CLAVE \\ Fibrilación auricular; \\ Tratamiento \\ anticoagulante; \\ Enfermedad \\ cerebrovascular
}

\begin{abstract}
Resumen
Introducción: La fibrilación auricular, la arritmia cardiaca más frecuente, incrementa el riesgo de ataque cerebrovascular cinco veces. La prevalencia en Colombia se estimó en 3,6\% en población mayor de 60 años (unos 180.000 pacientes).

Objetivo: Estimar la costo-efectividad de dabigatrán 110 y $150 \mathrm{mg}$ dos veces al día para el tratamiento de la fibrilación auricular no valvular en Colombia.

Métodos: Con perspectiva de tercer pagador (sistema de salud), se usó un modelo de Markov con ciclos de tres meses, seis estados de salud (y muerte): ataque cerebrovascular no discapacitante, accidente cerebrovascular discapacitante, infarto de miocardio; y dos eventos de transición: sangrado menor y mayor. Las probabilidades de transición y proporciones de eventos se extrajeron del ensayo RE-LY, las utilidades se obtuvieron de la literatura, en tanto que los costos se tomaron de bases de datos oficiales, en pesos colombianos, con tasa de descuento del $5 \%$, y un horizonte temporal de toda la vida (cerca de 20 años). El umbral fue tres veces el PIB per cápita (cerca de 45 millones de pesos).

Resultados: En comparación con warfarina, los pacientes tratados con dabigatrán 150 y $110 \mathrm{mg}$ ganaron, en promedio, 0,37 y 0,23 años de vida, respectivamente, o 0,55 y 0,43 años de vida ajustados por calidad (AVAC). La RCEl para dabigatrán $150 \mathrm{mg}$ fue $\$ 23.078 .506$ por AVAC ganado, mientras que para dabigatrán de $110 \mathrm{mg}$ fue de $\$ 34.186 .731$.

Conclusiones: Dabigatrán (ambas dosis), comparado con warfarina, es una alternativa costoefectiva para el tratamiento de la fibrilación auricular no valvular.

(c) 2015 Sociedad Colombiana de Cardiología y Cirugía Cardiovascular. Publicado por Elsevier España, S.L.U. Este es un artículo Open Access bajo la licencia CC BY-NC-ND (http:// creativecommons.org/licenses/by-nc-nd/4.0/).
\end{abstract}

\footnotetext{
* Autor para correspondencia.

Correo electrónico: diego.rosselli@gmail.com (D. Rosselli).
} 


\section{KEYWORDS}

Atrial fibrillation; Anticoagulant therapy; Cerebrovascular diseases
Cost-effectiveness of dabigatran compared with warfarin in the treatment of patients with non valvular atrial fibrillation in Colombia

\begin{abstract}
Introduction: Atrial fibrillation (AF), the most common cardiac rhythm disorder, increases the risk of stroke risk by 5 fold. AF prevalence in Colombia has been estimated in $3.6 \%$ in population age 60 or over (some 180000 patients).

Objective: The aim of this study was to estimate cost-effectiveness of dabigatran 110 and $150 \mathrm{mg}$ BID compared with warfarin as a therapy for non valvular AF in Colombian population. Methods: From a third-party payer perspective (Colombian health system) we used a threemonth cycle Markov model with 6 health states (and death): non-disabling stroke, disabling stroke, myocardial infarction and pulmonary embolism; two additional events were minor and mayor bleeding. Transition probabilities and proportion of events were extracted from the RELY trial; utilities were derived from the literature. Costs for medications and procedures were obtained from official government databases, all costs were in 2014 Colombian pesos $(1 \mathrm{USD}=2.000 \mathrm{COP})$. Annual discount rate was $5 \%$ and we used a life time horizon (close to 20 years, on average). Cost-effectiveness threshold was 3 times per capita GDP (around USD 22,500).

Results: Compared with warfarin, patients treated with dabigatran 150 and $110 \mathrm{mg}$ gained, on average 0.37 and 0.23 life-years respectively, or 0.55 and 0.43 QALYs. The ICER for dabigatran $150 \mathrm{mg}$ was USD 11,537 per QALY, and for dabigatran $110 \mathrm{mg}$ was 17,090 per QALY gained.

Conclusions: Dabigatran 150 and $110 \mathrm{mg}$, compared with warfarin - the standard therapy- are cost-effective therapies for ambulatory treatment of patients with non valvular AF.

(c) 2015 Sociedad Colombiana de Cardiología y Cirugía Cardiovascular. Published by Elsevier España, S.L.U. This is an open access article under the CC BY-NC-ND license (http:// creativecommons.org/licenses/by-nc-nd/4.0/).
\end{abstract}

\section{Introducción}

La fibrilación auricular es el trastorno del ritmo cardíaco más frecuente; en un hospital universitario de Colombia se realizó un estudio epidemiológico sobre su prevalencia. Con base en dicha información se puede extrapolar a la población general una prevalencia aproximada del 3,6\% en sujetos mayores de 60 años ${ }^{1}$. La fibrilación auricular se asocia con la aparición de insuficiencia cardíaca congestiva y de ataque cerebrovascular ${ }^{2}$. Ambas entidades están estrechamente relacionadas; a través de estudios analíticos de la cohorte de Framingham se estableció que el riesgo de presentar un accidente cerebrovascular teniendo fibrilación auricular es de 4,8 veces más que el de un paciente sin esta última ${ }^{3}$. Los costos de fibrilación auricular se han estudiado principalmente en países desarrollados, discriminados en costos directos e indirectos. Según la revisión sistemática de Wolowacz ${ }^{4}$, el costo directo anual en Estados Unidos de un paciente con fibrilación auricular es de 10.100 a 14.200 dólares, mientras que en Europa se ha estimado entre 450 y 3.000 euros. En Colombia hay un estudio que muestra que los costos de la atención de un ataque cerebrovascular son mayores en los pacientes con fibrilación auricular ${ }^{5}$. La terapia estándar para la fibrilación auricular no valvular persistente o permanente ha sido la warfarina, de acuerdo con las guías de práctica clínica ${ }^{6,7}$; sin embargo, en los últimos años se ha aprobado el uso de nuevos anticoagulantes orales, como dabigatrán, para la terapia antitrombótica, con la ventaja de no exigir un control especial del paciente con paraclínicos y tener una menor variabilidad de los niveles del medicamento según la alimentación y la interacción con otros medicamentos ${ }^{8}$.
El objetivo de este análisis fue establecer si la terapia con dabigatrán, en comparación con warfarina, es costoefectiva para el tratamiento de pacientes con fibrilación auricular no valvular en el contexto colombiano.

\section{Metodología}

Se utilizó un modelo de estados de transición de Markov, donde se incluyó la terapia estándar para fibrilación auricular (warfarina) y el inhibidor directo de la trombina, dabigatrán. Se contemplaron seis estados de acuerdo con el comportamiento clínico de la fibrilación auricular y su tratamiento antitrombótico. De los seis estados, dos fueron de transición (sangrado mayor y menor), mientras los otros cuatro contemplaban los eventos clínicos: accidente cerebrovascular leve no incapacitante y discapacitante, infarto agudo de miocardio y embolia pulmonar. El estado absorbente fue la muerte. El análisis del modelo se desarrolló desde la perspectiva de costos médicos directos para un tercero pagador, el sistema de salud colombiano. Se estimó un horizonte temporal de 20 años, que es similar a la expectativa de vida para estos pacientes en Colombia, teniendo en cuenta la edad de presentación de la enfermedad.

Los parámetros clínicos del modelo se tomaron de la literatura, en tanto que los costos de los eventos y de las terapias se extrajeron de las bases de datos del gobierno (Sismed para medicamentos, tarifas ISS 2001 para procedimientos).

Las probabilidades de transición entre los estados del modelo se obtuvieron a partir de las tasas de aparición de los eventos de: ataque cerebrovascular leve o no 
discapacitante, ataque cerebrovascular discapacitante, embolia pulmonar, infarto agudo de miocardio, sangrado menor y mayor y muerte del estudio RE-LY ${ }^{9}$. Las utilidades, en años de vida ajustados por calidad, o AVAC, una medida que pondera los estados de salud con valores entre 0 (el peor estado de salud posible, o la muerte) y 1 (un estado hipotético de salud plena), se obtuvieron de la literatura. La búsqueda se realizó en la base de datos Cost Efectiveness Analysis (CEA) registry de Tufts University ${ }^{10}$. Se encontraron todos los estudios que reportaran utilidades para los estados de salud de interés y posteriormente se promediaron las utilidades para cada uno de los estados de salud.

Para obtener los costos de warfarina y dabigatrán se utilizó la metodología propuesta por el Instituto de Evaluación de Tecnologías de Salud (IETS) ${ }^{11}$ y se estimaron en pesos colombianos de 2014. En esta, sugieren tomar la información de costos del canal de ventas hechas por los laboratorios a las instituciones de la lista nacional de los precios promedio para todos los medicamentos registrados en el país; en caso de que el medicamento estuviera regulado por la Comisión Nacional de Precios de Medicamentos y Dispositivos Médicos, se tomaba el precio máximo regulado.

Para la obtención de los eventos clínicos para cada estado y de la frecuencia y uso de los mismos se realizó un panel de expertos (conformado por un cardiólogo, un internista, dos geriatras, un neurólogo y un paciente con accidente cerebrovascular), y se siguieron los lineamientos de las guías de práctica clínica de la Sociedad Colombiana de Cardiología para la búsqueda de la literatura y su análisis posterior ${ }^{12}$. De esta forma se establecieron los paraclínicos, los procedimientos médicos y los medicamentos a costear. Los costos de cada evento clínico se extrajeron del manual tarifario del Instituto de Seguro Social (ISS) de 2001 con un incremento del $30 \%$ en el precio, por ser la tarifa más común de contratación entre pagadores y prestadores, y de uso regular en los estudios económicos. La tasa de descuento se ajustó al $5 \%$ para costos de medicamentos, eventos clínicos y utilidades.

Como desenlace principal se utilizó el AVAC y su razón de costo-efectividad incremental (RCEI) para dabigatrán $110 \mathrm{mg}$ y dabigatrán $150 \mathrm{mg}$, calculados a partir de la diferencia en los costos anuales contra la terapia estándar y la ganancia en AVAC por la terapia en relación con la warfarina. Siguiendo las recomendaciones del Instituto de Evaluación Tecnológica en Salud (IETS) ${ }^{13}$, el umbral de costo-efectividad empleado fue de hasta tres veces el PIB per cápita para Colombia (\$45.026.379).

Se realizaron diferentes tipos de análisis de sensibilidad a los resultados del caso base. Como primer ajuste, se modificó la tasa de descuento para los precios de los medicamentos, los costos de cada estado de salud y las utilidades. Las tasas de descuento sugeridas por el IETS son de $0,3,5$, 7 y $12 \%$. En segunda medida, el umbral de costo-efectividad fue modificado, pasando de tres veces a una vez el PIB per cápita. Por último, se utilizaron los costos mínimos y máximos de los estados clínicos de acuerdo con la variabilidad que puede presentar un paciente en el control de sus patologías. Finalmente, se hicieron varios escenarios modificando el horizonte temporal.

\section{Resultados}

El caso base, con un horizonte temporal de 20 añossimulando la expectativa de vida colombiana, a una tasa de descuento del $5 \%$ para costos y utilidades futuras, muestra que la ganancia total de AVAC para la terapia estándar (warfarina) es de 7,31 y su costo de $\$ 90.270 .760$. Para dabigatrán $150 \mathrm{mg}$ se mostró una ganancia adicional de 0,55AVAC (total de 7,86) y un costo total de la terapia de $\$ 103.041 .339$ (\$12.770.579 más que warfarina). En la dosis de $110 \mathrm{mg}$ cada $12 \mathrm{~h}$, dabigatrán obtuvo una ganancia adicional a warfarina de 0,43 AVAC (total de 7,73 AVAC) y un costo total de $\$ 104.801 .518$ (diferencia contra warfarina de $\$ 14.530 .758)$. La RCEI de dabigatrán $150 \mathrm{mg}$ es de $\$ 23.078 .506$ por AVAC ganado, mientras que para dabigatrán $110 \mathrm{mg}$ es de $\$ 34.186 .731$ por AVAC ganado. En la figura 1 se grafica la RCEI para dabigatrán en sus dos presentaciones en comparación con la terapia estándar. Parte de esta ganancia en AVAC se explica por mayor sobrevida, pero también por la reducción de eventos adversos, principalmente sangrados mayores.

Tabla 1 Resultado del análisis de costo-efectividad según las tasas de descuento de 0, 3,5, 7 y $12 \%$

\begin{tabular}{|c|c|c|c|c|c|c|c|}
\hline \multirow[t]{2}{*}{ Tasa de descuento } & \multirow[b]{2}{*}{ AVAC } & \multicolumn{2}{|c|}{ Warfarina } & \multicolumn{2}{|c|}{ Dabigatrán $150 \mathrm{mg}$} & \multicolumn{2}{|c|}{ Dabigatrán $110 \mathrm{mg}$} \\
\hline & & Costo & AVAC & Costo & AVAC & Costo & \\
\hline \multirow[t]{3}{*}{$0 \%$} & Valor & 10,65 & $\$ 152.826 .064$ & 11,59 & $\$ 177.841 .180$ & 11,36 & $\$ 180.745 .335$ \\
\hline & Diferencia & & & 0,93 & $\$ 25.015 .117$ & 0,70 & $\$ 27.919 .272$ \\
\hline & RCEI & & & $\$ 26.812 .329$ & $\$ 39.695 .748$ & & \\
\hline \multirow[t]{3}{*}{$3,5 \%$} & Valor & 8,11 & $\$ 103.364 .680$ & 8,75 & $\$ 119.979 .048$ & 8,60 & $\$ 122.003 .939$ \\
\hline & Diferencia & & & 0,64 & $\$ 16.614 .367$ & 0,49 & $\$ 18.639 .259$ \\
\hline & RCEI & & & $\$ 25.928 .645$ & $\$ 38.088 .527$ & & \\
\hline \multirow[t]{3}{*}{$7,5 \%$} & Valor & 6,43 & $\$ 73.275 .384$ & 6,89 & $\$ 85.041 .675$ & 6,78 & $\$ 86.520 .297$ \\
\hline & Diferencia & & & 0,46 & $\$ 11.766 .292$ & 0,36 & $\$ 13.244 .913$ \\
\hline & RCEI & & & $\$ 25.535 .279$ & $\$ 37.132 .810$ & & \\
\hline \multirow[t]{3}{*}{$12 \%$} & Valor & 4,87 & $\$ 48.174 .037$ & 5,18 & $\$ 55.750 .395$ & 5,12 & $\$ 56.761 .879$ \\
\hline & Diferencia & & & 0,31 & $\$ 7.576 .358$ & 0,24 & $\$ 8.587 .842$ \\
\hline & RCEI & & & $\$ 24.551 .446$ & $\$ 35.265 .386$ & & \\
\hline
\end{tabular}

AVAC: años de vida ajustados por calidad; RCEI: razón de costo-efectividad incremental. 
Tabla 2 Resultado del análisis de costo-efectividad ajustado por los valores mínimos y máximos de los estados clínicos

\begin{tabular}{|c|c|c|c|c|c|c|c|}
\hline & & \multicolumn{2}{|c|}{ Warfarina } & \multicolumn{2}{|c|}{ Dabigatrán 150 mg } & \multicolumn{2}{|c|}{ Dabigatrán 110 mg } \\
\hline & & AVAC & Costo & AVAC & Costo & AVAC & Costo \\
\hline \multirow[t]{3}{*}{ Valores mínimos } & Valor & 4,87 & $\$ 41.462 .486$ & 5,18 & $\$ 49.134 .179$ & 5,12 & $\$ 50.084 .863$ \\
\hline & Diferencia & & & 0,31 & $\$ 7.671 .693$ & 0,24 & $\$ 8.622 .377$ \\
\hline & RCEI & & & $\$ 24.860 .383$ & $\$ 35.407 .201$ & & \\
\hline \multirow[t]{3}{*}{ Valores máximos } & Valor & 4,87 & $\$ 64.944 .638$ & 5,18 & $\$ 70.388 .933$ & 5,12 & $\$ 71.495 .557$ \\
\hline & Diferencia & & & 0,31 & $\$ 5.444 .295$ & 0,24 & $\$ 6.550 .918$ \\
\hline & RCEI & & & $\$ 17.642 .422$ & $\$ 26.900 .898$ & & \\
\hline
\end{tabular}

AVAC: años de vida ajustados por calidad; RCEl: razón de costo-efectividad incremental.

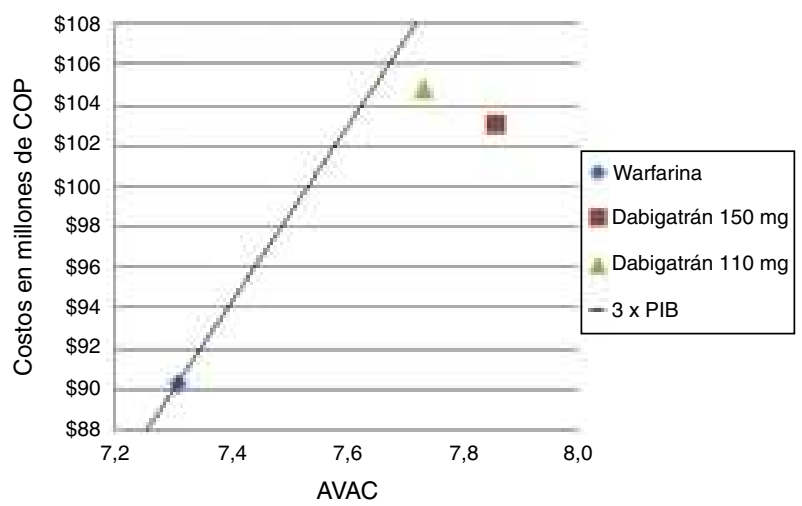

Figura 1 Gráfico de costo-efectividad de dabigatrán 110 y $150 \mathrm{mg}$. La línea corresponde al umbral de tres veces el PIB per cápita.

En la tabla 1 se muestra el resultado del análisis de sensibilidad para cada tasa de descuento empleada $(0,3,5,7$ y $12 \%$ ) de los costos totales de tratamiento y las utilidades.

Si se modificara el umbral de costo-efectividad ajustándolo a solo el PIB per cápita (\$15.008.793), ninguna de las dos presentaciones de dabigatrán sería costo-efectiva en el contexto colombiano.

Al utilizar los valores mínimos y máximos de cada estado de salud, varían los resultados en pequeña proporción, lo que sugiere que son la eficacia y la seguridad, más que los costos de los medicamentos y de las complicaciones, los factores que más influyen en el modelo. Los resultados se muestran en la tabla 2.

\section{Discusión}

Los resultados de la evaluación de costo-efectividad de dabigatrán, en el tratamiento de la fibrilación auricular no valvular en Colombia, muestran un incremento de los AVAC ganados con dabigatrán para los 2 regímenes de dosificación disponibles (150 y $110 \mathrm{mg}$ ), en comparación con warfarina. Esta ganancia en AVAC es atribuible a la disminución de la incidencia de desenlaces clínicos adversos tanto mortales como discapacitantes y, por ende, a un aumento de la sobrevida y calidad de vida de los pacientes con fibrilación auricular. La ganancia en AVAC de nuestro modelo es conservadora, en comparación con la descrita por Kongnakorn et al. ${ }^{14}$, que en su estudio de costo-efectividad encontraron una ganancia promedio en AVAC (combinando las dosis de 110 y $150 \mathrm{mg}$ ) de 0,11 con dabigatrán con respecto a warfarina, obteniendo así una RCEI de 7.585 euros.

Además de la efectividad a largo plazo de dabigatrán en la prevención de tromboembolia sistémica, la utilización de anticoagulantes orales directos podría tener ciertas ventajas respecto a la warfarina, ampliando la ventana terapéutica, sin la necesidad de realizar ajustes frecuentes de la dosificación ${ }^{15}$. La evaluación de los datos obtenidos sobre seguridad a largo plazo del estudio RELY-ABLE ${ }^{16}$, que siguió a 2.188 pacientes durante algo más de dosaños, no difiere de la reportada en el estudio original ${ }^{17}$.

Entre las fortalezas del estudio se encuentra la obtención de las probabilidades de transición entre los estados, a partir de las tasas de eventos presentadas en el estudio clínico $\mathrm{RE}-\mathrm{LY}^{9}$ y la utilización de datos sobre costos en Colombia. La aplicabilidad de los resultados dentro del sistema de salud colombiano facilitaría la toma de decisiones, al tiempo que permitiría la modificación de la distribución de recursos por parte de los pagadores destinados al tratamiento y la prevención de las complicaciones derivadas de la enfermedad.

\section{Responsabilidades éticas}

Protección de personas y animales. Los autores declaran que para esta investigación no se han realizado experimentos en seres humanos ni en animales.

Confidencialidad de los datos. Los autores declaran que en este artículo no aparecen datos de pacientes.

Derecho a la privacidad y consentimiento informado. Los autores declaran que en este artículo no aparecen datos de pacientes.

\section{Financiación}

Este trabajo recibió el apoyo económico de Böehringer Ingelheim.

\section{Conflicto de intereses}

Ludy Parada es actualmente funcionaria de Boehringer Ingelheim. Diego Rosselli ha sido consultor y conferencista para Boehringer Ingelheim. El resto de los autores declara no tener ningún conflicto de intereses. 


\section{Bibliografía}

1. Rosselli D, Rodríguez AJ, García AA, Rueda JD. Prevalencia de fibrilación auricular en un hospital universitario colombiano. Rev Colomb Cardiol. 2013;20:383-5.

2. Krahn AD, Manfreda J, Tate RB, Mathewson FA, Cuddy TE. The natural history of atrial fibrillation: incidence, risk factors, and prognosis in the Manitoba Follow-Up Study. Am J Med. 1995; 98:476-84.

3. Wolf PA, Abbott RD, Kannel WB. Atrial fibrillation as an independent risk factor for stroke: The Framingham Study. Stroke. 1991;22:983-8.

4. Wolowacz SE, Samuel M, Brennan VK, Jasso-Mosqueda J-G, van Gelder IC. The cost of illness of atrial fibrillation: A systematic review of the recent literature. Europace. 2011;13:1375-85.

5. Castañeda C, Rueda JD, Díaz CE, Coral J, Rueda MC, Rosselli D. Análisis de costos de infarto cerebral agudo con o sin fibrilación auricular. Acta Neurol Colomb. 2014;30:78-82.

6. Fuster V, Rydén LE, Asinger RW, Cannom DS, Crijns HJ, Frye RL, et al. ACC/AHA/ESC guidelines for the management of patients with atrial fibrillation: executive summary. J Am Coll Cardiol. 2001;38:1231-66.

7. Guidelines for medical treatment for stroke prevention. American College of Physicians. Ann Intern Med. 1994;121:54-5.

8. Hylek EM. Anticoagulation therapy for atrial fibrillation. Semin Thromb Hemost. 2013;39:147-52.

9. Connolly S, Ezekowitz M, Yusuf S, Oldgren J, Pogue J, Reilly P, et al. Dabigatran versus warfarin in patients with atrial fibrillation. N Engl J Med. 2009;361:1139-51.

10. Tufts University Cost Efectiveness Analysis (CEA) Registry [consultado 7 Nov 2014]. Disponible en: https: / / research.tuftsnemc.org/cear4/
11. Instituto de Evaluación Tecnológica en Salud-IETS. Manual metodológico para la elaboración de evaluaciones de efectividad, seguridad y validez diagnóstica de tecnologías en salud [consultado 8 Ene 2015). Disponible en: http://www.iets.org.co/ Manuales/Manuales/Manual\%20EyS\%20\%20web_30\%20sep.pdf

12. Sociedad Colombiana de Cardiología y Cirugía Cardiovascular. Guías de diagnóstico y tratamiento de la fibrilación auricular 2012 [consultado 18 Oct 2014]. Disponible en: http://scc.org.co/blog/2012/08/guias-de-diagnosticoy-tratamiento-de-la-fibrilacion-auricular/

13. Instituto de Evaluación Tecnológica en Salud. Manual para la elaboración de evaluaciones económicas en salud. Bogotá D.C.: IETS; 2014 [consultado 25 May 2015]. Disponible en: http:// www. iets.org.co/Manuales/Manuales/Manual\%20evaluacio\%CC \%81n\%20econo\%CC\%81mica\%20web\%2030\%20sep.pdf

14. Kongnakorn T, Lanitis T, Annemans L, Thijs V, Goethals M, Marbaix $\mathrm{S}$, et al. Stroke and systemic embolism prevention in patients with atrial fibrillation in Belgium: Comparative cost effectiveness of new oral anticoagulants and warfarin. Clin Drug Investig. 2015;35:109-19.

15. Mullein N, Cei M, Vitale J, Fontanella A, Dentali F. Are direct oral anticoagulants effective in reducing systemic embolism in patients with atrial fibrillation? A systematic review and metaanalysis of the literature. Int J Cardiol. 2015;180:192-5.

16. Connolly SJ, Wallentin L, Ezekowitz MD, Eikelboom J, Oldgren J, Reilly PA, et al. The long term multicenter observational study of dabigatran treatment in patients with atrial fibrillation (RELYABLE) Study Circulation. 2013;128:237-43.

17. FDA drug safety communication: Update on the risk for serious bleeding events with the anticoagulant Pradaxa (dabigatran) [consultado 12 Feb 2015]. Disponible en: http://www.fda.gov/ Drugs/DrugSafety/ucm326580.htm 